\title{
Fear and Perception of People to Visit Dentists during COVID-19 Pandemic and Their Suggestions
}

\author{
Muhammad Mansoor Majeed ${ }^{1}$ Zarah Sidiqqui ${ }^{2} \quad$ Muhammad Uzair $^{3} \quad$ Azam Shahzad $^{4}$ Sara Rafique ${ }^{5}$ \\ Shoaib Durrani 6
}

${ }^{1}$ Department of Oral Biology, Altamash Institute of Dental Medicine, Karachi, Pakistan

2 Department of Prosthodontics, Dow University of Health Sciences, Karachi, Pakistan

${ }^{3}$ Department of Orthodontics, Baqai Dental College, Karachi, Pakistan

${ }^{4}$ Department of Dental Materials, Shahida Islam Dental College, Lodhran, Pakistan

${ }^{5}$ Department of Physiology, Jinnah Medical and Dental College,

Karachi, Pakistan

${ }^{6}$ Durrani Dental Surgery, Pakistan Dental Association (Karachi

Chapter), Karachi, Pakistan

Eur J Gen Dent 2021;10:129-134.
Address for correspondence Muhammad Mansoor Majeed, BDS, MDS, Department of Oral Biology, Altamash Institute of Dental Medicine, Karachi, Sindh 75500, Pakistan

(e-mail: mmansoormajeed@gmail.com).

\begin{abstract}
Keywords

- COVID-19

- patients

- fear

- anxiety

- dentists

Objectives This study was performed to determine patients' fear and perception of visiting dentists during the COVID-19 pandemic.

Materials and Methods This cross-sectional study was performed online using Google forms. Participants included individuals who had been to dental clinics for treatment. The data of 407 participants were analyzed. SPSS 21 was used for data entry and analysis. Demographic variables were expressed in numbers and percentages. The Chi-squared test was applied to analyze the difference between the demographic variables and fear among genders, age, and level of education.

Results Due to the coronavirus's contagious nature, fear is prevalent among a significant number of patients $\left(x^{2}=31.373, p<0.001\right)$. Females are more afraid to visit the dentist than males $\left(x^{2}=7.065, p=0.008\right)$. The majority of the patients are not willing to go for dental treatment other than an emergency. Around two-thirds of the participants reported that their visit to the dentist during the current pandemic was reduced.

Conclusion Due to the fear of COVID-19, patients are not willing to visit dentists for treatments. Therefore, dentists should build confidence by ensuring cross-infection control.
\end{abstract}

\section{Introduction}

COVID-19 came under attention in December 2019 and was declared a pandemic in March 2020 by the World Health Organization (WHO). Inexplicable cases of pneumonia appeared in Wuhan, China, whose rapid spread has disordered many countries worldwide. ${ }^{1}$

Due to the contagious nature of this disease, governments imposed lockdown, and social activities were ceased. Variations to the typical traditions of life can make individuals feel
DOI https://doi.org/ $10.1055 / \mathrm{s}-0041-1736377$. ISSN 2278-9626.

\footnotetext{
(c) 2021. European Journal of General Dentistry. All rights reserved. This is an open access article published by Thieme under the terms of the Creative Commons Attribution-NonDerivative-NonCommercial-License, permitting copying and reproduction so long as the original work is given appropriate credit. Contents may not be used for commercial purposes, or adapted, remixed, transformed or built upon. (https://creativecommons.org/ licenses/by-nc-nd/4.0/)

Thieme Medical and Scientific Publishers Pvt. Ltd., A-12, 2nd Floor, Sector 2, Noida-201301 UP, India
} 
worried and insecure spirits of being perilous can be related to the disease-fear contagion link; for instance, not knowing the source or development of the ailment and consequences, rumors, and misrepresentation can lead to intolerance against persons of specific descent. ${ }^{2}$

The COVID-19 pandemic has adversely affected the global economy, national healthcare systems, and pharmaceutical firms' income, revenue, and operations. The harmful impacts of the COVID-19 pandemic encompass high rates of infections and death, government and individuals' financial burden, stress, and fear of uncertainty. ${ }^{3}$ Due to COVID-19, not only healthcare workers are under psychological stress, ${ }^{4,5}$ but those seeking treatment or in need of healthcare facilities and patients with varying diseases suffer from anxiety, depression, and fear. ${ }^{6}$ A study on pregnant women reported that $83 \%$ of women did not worry about their health, but during the current pandemic, over half of the women were worried. ${ }^{7}$ Another study on pregnant women stated that $84.6 \%$ of the females were afraid of COVID-19 infection and reported a mean fear level of $5.86 \pm 3.12$ on a scale of 1 to 10 . Women who perceived that COVID-19 can affect the child had a significantly higher level of Generalized Anxiety and Depression (GAD) scores. Similarly, women who were afraid of COVID-19 infection had significantly higher GAD scores. ${ }^{8}$ Similarly, cancer patients also experienced stress and anxiety due to this highly contagious disease. ${ }^{9}$ Likewise, patients seeking dental treatment are also afraid to visit dentists. ${ }^{10,11}$

It has always been challenging for the dental setup staff to maintain cross-infection control and, mainly, during dental procedures that produce aerosols. Due to the nature of dental care surroundings, the hazard of cross-infection is elevated in patients and dentists, and their coworkers. ${ }^{12}$ Studies have reported that during dental treatment, fear and anxiety have crossed another level among dentists and dental patients. ${ }^{10,13}$ As the chances of spreading this contagious virus are much higher from the dental offices, many dental associations limited the treatment to emergencies. ${ }^{14,15}$ By maintaining proper cross-infection control, the use of personnel protective equipment (PPE), and following SOPs, the spread can be controlled. Moreover, studies have reported that saliva exhibits high sensitivity and specificity for the detection of SARSCOV-2. ${ }^{16}$ Therefore, the dentist can check and detect this novel coronavirus before starting the procedure. ${ }^{17}$ A previous study has also reported that uncertainty prevails among the patients to visit the dentist for their treatment because of this pandemic. ${ }^{10}$ However, little data are available about patients' perception and their anxiety regarding their dental treatment due to COVID-19.

There is a need to understand and tackle the increasing psychological dilemma among different people during the COVID-19 pandemic. Hence, this study aimed to examine the effect of the COVID-19 pandemic on patient's dental care perceptions and concerns.

\section{Materials and Methods}

This online cross-sectional study was performed months after COVID-19 was declared a pandemic by the WHO.
Patients undergoing dental treatment from different parts of the world were included in the study via the snowball sampling technique. A self-designed, content-validated questionnaire was used, which consisted of three parts: Demographic characteristics were gender, age, and educational level. A self-reported question was whether the participant was afraid of COVID-19 or not, patients' perception of the visit to the dentist, and change of their mindset due to COVID-19. One optional request from the patients for their suggestions to the dentist was also offered in the survey form. The study was approved by the ethics and review committee of the Altamash Institute of Dental Medicine (AIDM/EC/04/2020/ 05). SPSS 21 was used for data entry and analysis. Descriptive statistics of the responses were expressed as percentages. Categorical variables were stated as frequencies and percentages. The Chi-square test was applied to find out significance levels of demographic characteristics. A p-value of less than 0.05 was considered significant.

\section{Results}

In the current study, the data of 429 individuals were collected. Due to the discrepancies, 22 forms were excluded from the analysis. Out of 407 participants, a significantly higher number of the individuals 260 (63.88\%) reported that they were afraid of COVID-19 $\left(X^{2}=31.37, p<0.001\right)$. A substantially higher number of females reported they were afraid of COVID-19 than males $\left(X^{2}=7.065, p<0.008\right)$. No significant difference between the two age groups and three different education levels was observed $(p>0.05)$ ( $~-$ Table1).

In the current study, due to fear, most participants avoided routine dental treatment. We observed that 74 (18.18\%) said during COVID-19, no dental treatment should have opted, and $272(66.83 \%)$ stated that only emergency dental treatment could be obtained. A limited number of the patients agreed to have other dental treatments such as dental fillings, root canal treatment, and extraction ( $\mathbf{- T a b l e ~} \mathbf{2}$ ).

The majority (68.30\%) of the participants indicated that their frequency of visiting the dentist was reduced ( - Fig. 1).

\section{Discussion}

The current pandemic situation has scared the individuals to visit the clinic, specifically patients who have been to dentists. The current pandemic has changed the general public's psychology due to the coronavirus's highly contagious nature. Fear and anxiety due to COVID-19 has been reported in a different group of people in several studies. Moreover, most of them are reluctant to visit dental clinics for their routine visit or dental treatment. Various studies have indicated the fear and anxiety among patients in perceiving dental treatment. This fear is not only because of the fear of the spread of COVID-19 but also due to the costs of personnel protective equipment and new products. In addition, the government policies have increased the expenses of the dentist; therefore, the mindset of patients has changed. ${ }^{10,18}$

In the current study, we observed that females were more afraid of COVID-19 than males. These findings are similar to 
Table 1 Demographics and fear of COVID-19 among the study participants

\begin{tabular}{|c|c|c|c|c|c|c|}
\hline \multicolumn{2}{|c|}{ Are you afraid of COVID-19? } & Number (\%) & Yes & No & $x^{2}$ & $p$-Value \\
\hline \multirow[t]{3}{*}{ Gender } & Male & $226(55.52)$ & $132(58.40)$ & $94(41.59)$ & \multirow[t]{2}{*}{7.065} & \multirow[t]{2}{*}{0.008} \\
\hline & Female & $181(44.47)$ & $128(70.71)$ & $53(29.28)$ & & \\
\hline & Total & 407 & $260(63.88)$ & $147(36.11)$ & 31.373 & $<0.001$ \\
\hline \multirow[t]{2}{*}{ Age group } & Up to 40 years & $262(64.37)$ & $167(63.74)$ & $95(36.25)$ & \multirow[t]{2}{*}{006} & \multirow[t]{2}{*}{0.936} \\
\hline & 41 and above & $145(35.62)$ & $93(64.13)$ & $52(35.86)$ & & \\
\hline \multirow[t]{3}{*}{ Education } & Undergraduate & $40(9.82)$ & $21(52.5)$ & $19(47.5)$ & \multirow[t]{3}{*}{2.492} & \\
\hline & Graduate & $181(44.47)$ & $118(63.44)$ & $63(34.80)$ & & \\
\hline & Postgraduate & $186(45.70)$ & $121(65.05)$ & $65(34.94)$ & & \\
\hline
\end{tabular}

Table 2 Which treatment option could be opted during the COVID-19 pandemic?

\begin{tabular}{|l|l|}
\hline Treatment option & N (\%) \\
\hline No treatment at all & $74(18.18)$ \\
\hline Only emergency treatment & $272(66.83)$ \\
\hline Only dental checkup & $204(50.12)$ \\
\hline Dental filling & $131(32.18)$ \\
\hline Root canal treatment & $141(34.64)$ \\
\hline Extraction & $145(35.62)$ \\
\hline Orthodontic treatment & $98(24.07)$ \\
\hline Teeth scaling & $96(23.58)$ \\
\hline Any treatment can be done & $9(2.21)$ \\
\hline
\end{tabular}

studies performed in different parts of the world. ${ }^{10,19}$ Not only female patients but female healthcare professionals reported anxiety, fear, and depression.
In the current study, 272 (66.83\%) patients stated they would get a dental treatment only in the case of an emergency, and similar findings were also reported in a survey conducted during the early phase of COVID-19 in Brazil. ${ }^{20}$

In our opinion, the anxiety and fear among the patients regarding dental treatment and visits to dental clinics is created by the dentists by speaking negatively about dentistry. Dentists have openly reported about the risk in their profession. On social media, they were holding posters "No work-Stay home," and slogans such as "no one can die without dental treatment" have pushed the profession decades back. Furthermore, speaking negatively of the profession has not only changed the perceptions of old dental patients but also scared the new patients. This has also made a big dent in the finances of the dentists and has changed the mindset of dental patients, and it will take some time before things improve. ${ }^{10}$

In the current study, it was observed that routine dental care visits were significantly reduced. This will not only bring a drastic effect on the oral health of patients but will also be

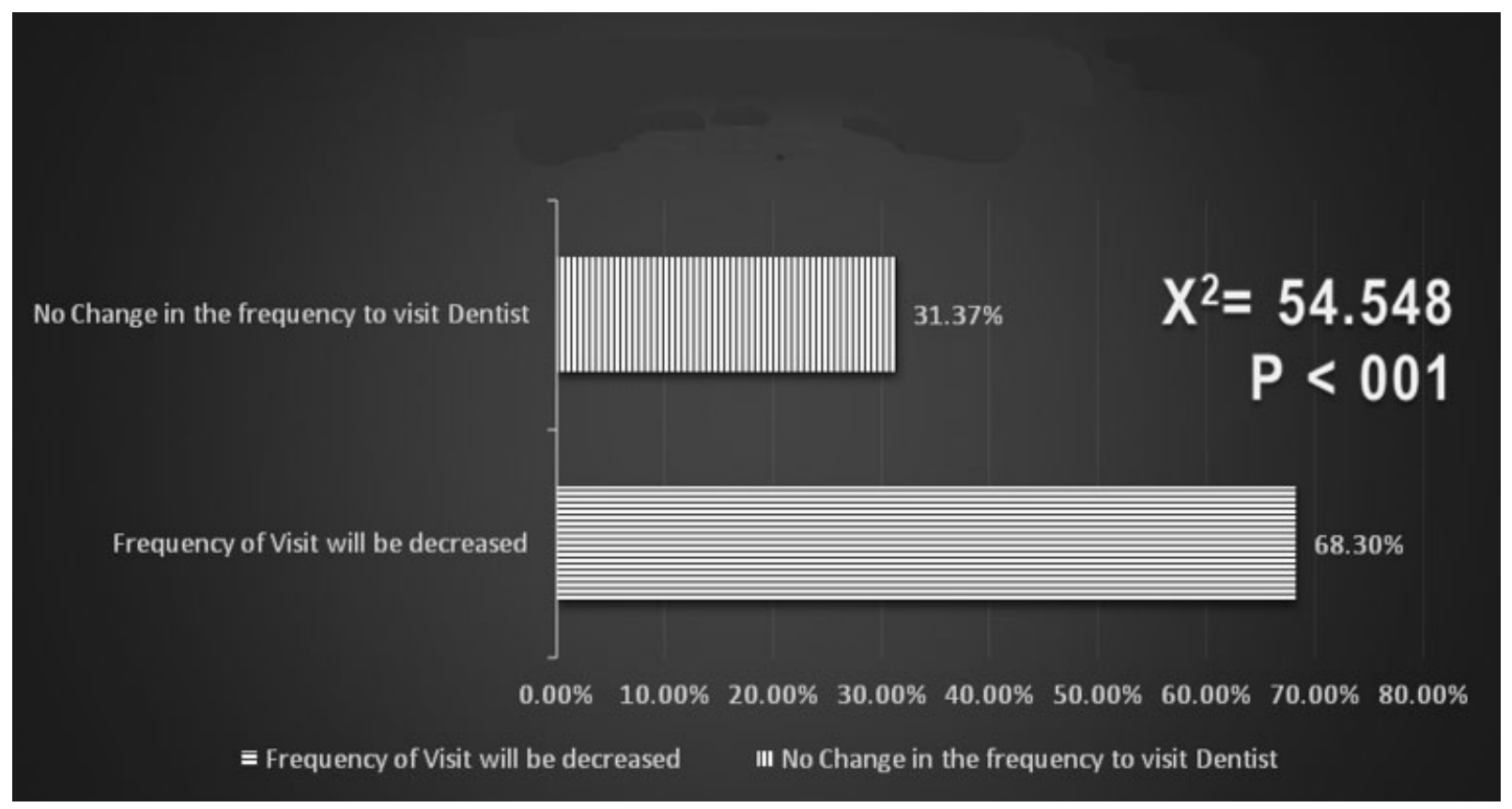

Fig. 1 Change in the routine to visit the dentist due to COVID-19 pandemic. 
devastating for the dentists economically. These conclusions are also similar to the studies published previously. For example, a Polish study observed a significant decrease in patients since the emergence of COVID-19, a study performed in Japan reported a decline in the number of patients. ${ }^{21,22}$ Moreover, a nationwide survey performed in Pakistan reported that dental employers faced incredible challenges in managing the expenses due to the few numbers of patients. $^{23}$

In the existing study, most individuals stated that they would only opt for emergency dental treatments during the outbreak of the COVID 19. It has been advised by many dental associations and authorities to limit the dental community to avoid performing any elective dental procedures and provide emergency treatment only. Moreover, due to the lockdown where all aspects of life have been shut down, dental colleges and teaching hospitals are also not operating or providing only emergency dental treatments. ${ }^{24}$

A study has reported that the general public and particularly dental patients are aware of the protocols, and they emphasize following SOPs and proper disinfection of the dental chair and surgery after every dental treatment. ${ }^{25}$ In the current study, similar suggestions were given by the patients. A previous study also reported a significant and positive attitude of dental health professionals toward disinfection regarding the COVID-19 pandemic. $^{26,27}$

The coronavirus has majorly impacted the psychology and perceptions of patients seeking dental treatment. The majority of patients are anxious about visiting the dental clinic amid this pandemic and contracting the deadly virus. Techniques for cross-infection control should be implemented and the trust of patients should be built. Moreover, the implementation of teledentistry will help to control the spread of COVID-19. ${ }^{28}$

\section{Limitations}

Due to the lockdown, it was difficult to collect the data in personnel. Moreover, online data collection may have some biases. Only those patients were included in the study who were tech-friendly or had access to the specific messaging platform.

In the current study, we asked for suggestions. These patients' suggestions and requests may help the dentists understand their perceptions and demands and eventually build confidence and optimism in them.

S1: We care about you as you care for us; please stay safe. Maybe a doctor will treat someone positive for COVID-19, and from him, the doctor itself gets the disease.

S2: Build a mobile dental treatment clinic on a minivan and during this time give dental treatment at their doorstep after following all protocols.

S3: Keep a hygienic, clean clinic with a clean and healthy environment.

S4: Dental hospitals and clinics should remain open during this pandemic because sometimes these dental pains are extreme and intolerable that may require procedural intervention. On the contrary, dentists must ensure and take robust and extreme measures to keep themselves and their patients protected from COVID-19. Constant sanitization of the entire clinic and absolute sterilization of the medical and PPE is a must whenever a patient is attended.

S6: Doctors and clinics should follow all protocols during treatments to avoid the spread of COVID-19.

S7: These are challenging times, and our doctors, paramedic staff, law enforcement agencies, security staff, and IT guys are doing commendable services to the community. Rather than charging back to patients, such a well-known setup should take extra precautionary steps and maintain hygiene standards to lead the example. Also, cater to emergency treatment only with all safety protocols.

S8: Do not overcharge during this time of crisis.

S9: I guess the dentists should be available on calls as $90 \%$ of the people reach dentists via friends or family references.

S10: Dentists should take care of themselves too, they can assist in some urgency over the phone or if a patient is in pain or need treatment; the doctor must go through all safety procedures before treating the patient.

S11: To use proper PPEs according to the WHO standards. Try to use disposable instruments as much as possible; use aerosol evacuators.

S12: Like all other medical treatments during the pandemic, optional dental treatments should be deferred. However, dentists should now prepare to handle the challenges of the post-pandemic phase.

S13: I think patients have not only a fear of catching COVID-19 by getting outside but also the inflation and low-income generation due to lockdown, and people are more likely to spend money on their basic needs and emergency treatments. However, provided with due safety measures for COVID-19 by dentists or healthcare professionals, people will gain the confidence to get treatment. The government has to play its role to normalize the situation and not create panic in the society that will result in overall improved economic conditions, which in turn will improve the financial condition of dentists as well.

S14: Stay home stay safe, only attend to patients in case of emergency.

S15: The doctor should fully understand the safety precautions regarding this virus. They should wear a proper dress to avoid risk factors. Due to the present situation, I suggest that some new techniques be adopted or added to the treatment, such as magnifying tools and the latest camera techniques to avoid close contact with patients.

S16: (1) Dentists should check patients for COVID-19 before starting the treatment. (2) Patients should 
be given an appointment in a 1-hour gap before the next patient arrives for treatment. (3) The doctor and assistant should use a face mask to provide protection and safety to the patient. (4) Use hygienic tools and sanitizer to satisfy the patient and follow medical protocols.

S17: Kindly provide all necessary information regarding COVID-19 at the entrance of dental clinics and how it can spread via dental procedures, and which dental procedures are contraindicated during this pandemic.

S18: Routine dental procedures can take a break due to the pandemic, but the dentist should keep in telecom touch with their patients to keep up with any emergency treatments. Dental pain will not stop itself from occurring in a patient due to the pandemic.

S19: Limit the practice to only emergency cases until the COVID-19 curve goes below a certain threshold.

S20: Only patients with an appointment should be attended to. No one to be allowed in the waiting area. Wear PEP. Install sanitizer machines in dental clinics.

S21: Patients should visit dentists in the extreme condition of pain (when the pain is unbearable). Dentists should adopt all necessary precautions and measures as advised by experts/government for themselves, staff, and the patients, to avoid infection of patients, dentists, and staff from COVID-19. All the best.

\section{Conclusion}

High anxiety and fear were reported among patients to visit the dental clinic in this pandemic. Patients should be assured that proper sanitization protocols have been followed by the clinic to build their trust and reduce their fear of contracting the disease by visiting the clinic.

\section{Authors' Contributions}

M.M. was the principal investigator, conceived the study, supervised the project, and is responsible for the integrity of the research. Comprehensively contributed to data collection, statistical work, writing of the manuscript, critical revision, and approval of the final draft. S.Z. contributed to initial draft writing, literature search, data collection, and final approval of the manuscript. U.M. contributed to study design, data collection, critical revision, and final approval of the manuscript. S.A. contributed to data collection, write-up, and approval of the final draft. R.S. contributed to write-up, statistics, tables, and figures, and final approval of the manuscript. D.S. contributed to write-up, statistical analysis, critical revision, and final approval of the manuscript.

\section{Conflict of Interest}

None declared.

\section{Acknowledgments}

We would like to acknowledge Dr. Muhammad Munir Hamirani, Professor of Psychiatry, for his valuable suggestions and guidance. We acknowledge the support of the ethics and review committee of Altamash Institute of Dental Medicine, Karachi, Pakistan. We would also like to thank all the respected participants.

\section{References}

1 Sohrabi C, Alsafi Z, O'Neill N, et al. World Health Organization declares global emergency: a review of the 2019 novel coronavirus (COVID-19). Int J Surg 2020;76:71-76

2 Usher K, Bhullar N, Jackson D. Life in the pandemic: social isolation and mental health. J Clin Nurs 2020;29(1516):2756-2757

3 Shreffler J, Petrey J, Huecker M. The impact of COVID-19 on healthcare worker wellness: a scoping review. West J Emerg Med 2020;21(05):1059-1066

4 Alwani SS, Majeed MM, Ramzan Z, Rauf S, Syed MS, Shah SH, Hirwani MZ, Hamirani F. Evaluation of knowledge, practices, attitude, and anxiety of nurses towards COVID-19 during the current outbreak in Karachi, Pakistan. Pakistan Journal of Public Health 2020;10(02):82-90

5 Majeed MM, Saleem Z, Sarwar H, Ramzan Z, Iqbal SN, Naeem MM. The psychology of coronavirus fear: are dentists of pakistan suffering from corona-phobia? J Pak Dent Assoc 2021;30(01): 1-6. Doi: 10.25301/JPDA.301.1

6 Lal A, Sanaullah A, M Saleem MK, Ahmed N, Maqsood A, Ahmed N. Psychological distress among adults in home confinement in the midst of COVID-19 outbreak. Eur J Dent 2020;14(S 01): S27-S33

7 Corbett GA, Milne SJ, Hehir MP, Lindow SW, O'connell MP. Health anxiety and behavioural changes of pregnant women during the COVID-19 pandemic. Eur J Obstet Gynecol Reprod Biol 2020; 249:96-97

8 Hossain N, Sameul M, Sandeep R, Imtiaz S, Zaheer S. Perceptions, generalized anxiety and fears of pregnant women about corona virus infection in the heart of pandemic. 2020, Preprint (Version 1). doi:10.21203/rs.3.rs-32235/v1. Available at: https://www.researchsquare.com/article/rs-32235/v1 Last accessed on July 15, 2021

9 Hoda W, Bharati SJ, Kumar A, Choudhary P, Shankar A. Sociocultural and economic impact of corona virus on cancer patients, caregivers and survivors. Asian Pacific Journal of Cancer Care 2020;5(S1):171-173

10 Majeed MM, Siddiqui Z, Tabbasum U, Sarwar H, Minhas S, Bhatia MR. Psychological barriers among dental patients during the COVID-19 crisis. Rawal Med J 2021;46(01):7-10

11 Pylińska-Dąbrowska D, Starzyńska A, Cubała WJ, Ragin K, Alterio D, Jereczek-Fossa BA. Psychological functioning of patients undergoing oral surgery procedures during the regime related with SARS-CoV-2 pandemic. J Clin Med 2020;9(10):3344

12 Samaranayake LP, Peiris M. Severe acute respiratory syndrome and dentistry: a retrospective view. J Am Dent Assoc 2004;135 (09):1292-1302

13 Salim Z, Shaikh AH, Ramzan Z, Bhatia MR, Tabassum U, Majeed MM. A comparative study to evaluate COVID-19 related anxiety and fear among medical doctors and dental surgeons. Ethiop Med J 2021;59(02):91-99

14 Coulthard P. Dentistry and coronavirus (COVID-19) - moral decision-making. Br Dent J 2020;228(07):503-505

15 Imran E, Khurshid Z, Adanir N, Ashi H, Almarzouki N, Baeshen HA. Dental practitioners' knowledge, attitude and practices for mouthwash use amidst the COVID-19 pandemic. Risk Manag Healthc Policy 2021;14:605-618

16 Warsi I, Khurshid Z, Shazam H, et al. Saliva exhibits high sensitivity and specificity for the detection of SARS-COV-2. Diseases 2021;9(02):38

17 Khurshid Z, Asiri FYI, Al Wadaani H. Human saliva: non-invasive fluid for detecting novel coronavirus (2019-nCoV). Int J Environ Res Public Health 2020;17(07):2225 
18 Guo H, Zhou Y, Liu X, Tan J. The impact of the COVID-19 epidemic on the utilization of emergency dental services. J Dent Sci 2020;15 (04):564-567

19 Moghanibashi-Mansourieh A. Assessing the anxiety level of Iranian general population during COVID-19 outbreak. Asian J Psychiatr 2020;51:102076

20 Peloso RM, Pini NIP, Sundfeld Neto D, et al. How does the quarantine resulting from COVID-19 impact dental appointments and patient anxiety levels? Braz Oral Res 2020;34:e84

21 Tysiąc-Miśta M, Dziedzic A. The attitudes and professional approaches of dental practitioners during the COVID-19 outbreak in Poland: a cross-sectional survey. Int J Environ Res Public Health 2020;17(13):4703

22 Nardone M, Cordone A, Petti S. Occupational COVID-19 risk to dental staff working in a public dental unit in the outbreak epicenter. Oral Dis 202110.1111/odi.13632

23 Sarwar H, Qureshi ND, Fatima S, Naeem MM, Inayat A. A nationwide survey on financial impact of COVID-19 on employers of private dental practices of Pakistan. JPDA 2020;29(04):172-178
24 Majeed MM, Durrani MS, Bashir MB, Ahmed M. COVID-19 and dental education in Pakistan. J Coll Physicians Surg Pak 2020;30 (10):115-117

25 Ahmed MA, Jouhar R, Adnan S, Ahmed N, Ghazal T, Adanir N. Evaluation of patient's knowledge, attitude, and practice of crossinfection control in dentistry during COVID-19 pandemic. Eur J Dent 2020;14(S 01):S1-S6

26 Sarfaraz S, Shabbir J, Mudasser MA, et al. Knowledge and attitude of dental practitioners related to disinfection during the COVID19 pandemic. Healthcare (Basel). 2020;8(03):23210.3390/ healthcare8030232

27 Farook FF, Mohamed Nuzaim MN, Taha Ababneh K, Alshammari A, Alkadi L. COVID-19 pandemic: oral health challenges and recommendations. Eur J Dent 2020;14(S 01)S165-S170

28 Abbas B, Wajahat M, Saleem Z, Imran E, Sajjad M, Khurshid Z. Role of teledentistry in COVID-19 pandemic: a nationwide comparative analysis among dental professionals. Eur J Dent 2020; 14(S 01):S116-S122 